\title{
DISTRIBUTED HIL SIMULATION OF MECHATRONIC SYSTEMS APPLIED TO AN AGRICULTURAL MACHINE
}

\section{Mauro Cesar Zanella, Ralf Stolpe}

\begin{abstract}
This paper presents an approach to a new software platform for distributed hardware-in-the-loop simulation applied to mechatronic systems.

An agricultural machine is presented as an example of a complex hierarchical mechatronic system. The decomposition of this machine into a hierarchical function-oriented form is shown in detail. To evaluate the communication and signal propagation as well as the I/O behaviour of a part of the complete system, a hardware-in-the-loop simulation of an active wheel suspension was implemented. The software approach to HILS is designed to support a hierarchical distributed control algorithm with the possibility of handling errors and dynamic coupling at run-time.
\end{abstract}

\section{Introduction}

Mechatronic systems consist of components from different disciplines, such as mechanical engineering, electrical engineering, and information science; each of them contributing their own specific functions to the generation of the utility. The concept of function in mechatronics can in most cases be seen as a synonym for the desired controlled motion behaviour of a system. A basic feature of mechatronic design is its decomposition into smaller subsystems based on the different functions of the system components. In addition to the mechanical supporting structure, actuators, sensors, and components are employed to put the mechatronic functions into effect (Honekamp 1997).

A proposal for the structuring of mechatronic systems as a basis for a generalized design method is presented in (Honekamp 1997). The approach discussed is a combination of hierarchical and modular organization with a distinction between the mechatronic system components. On the lowest hierarchical level, physically related sensors, actuators, mechanical structures, and information-processing units are combined to make up Mechatronic Function Modules (MFM). Two or more MFMs form an Autonomous Mechatronic System (AMS) which, in contrast to an MFM, excludes sensors or actuators. The highest hierarchical level is occupied by a combination of two or more AMSs to make up a Cross-linked Mechatronic System (CMS). 
Inside a CMS, the AMSs provide only passive mechanical coupling with one another; and are interconnected by means of informational devices.

The information-processing unit of the entire system can be implemented by means of a parallel software system. In this case each of the MFMs is equipped with a local information-processing unit made up of hard- and software. The MFMs (including information processing) can be tested separately by means of the hardware-in-theloop simulation technique. For this purpose the subsystem is embedded in a realistic environment which interacts with the subsystem as if the real operational environment were present. This paper will introduce a new approach to support real-time information processing in mechatronic systems.

The decomposition already mentioned and the new approach will then be exemplified by an agricultural machine, and we will give an overview of the complete machine with the HILS hardware.

Due to the large number of sensors and actuators in the machine, ECUs will been distributed along it with the propose to interface $\mathrm{I} / \mathrm{Os}$ with the control hardware. Communication with the ECUs will be effected via a CANbus.

To evaluate the ECUs, I/O and software as well as the time delay (sampling/setting signals and communication) of the system, a partial simulation of the agricultural machine has been implemented in the laboratory.

We will then present a novel concept of a software platform allowing a flexible distributed HIL simulation of the complete agricultural machine with error-handling at run-time. The main features of this software platform will be explained, as will be its elements. The concept will be implemented in three consecutive stages. The first and the second are an off-line simulation, one with one processor and the other, with two processors. The third stage is an HILS with two processors.

\section{Mechatronic Function Module (MFM) and Autonomous Mechatronic System (AMS), Exemplified by an Agricultural Machine}

The MFM is the lowest level of the hierarchical structure of a mechatronic system. It has sensors and actuators as its main features and comprises also passive mechanical couplings. The information-processing of the control has to be quasi-continuous and under hard real-time conditions. Security and emergency mechanims also have to be provided for.

As an example of MFMs, we use a wheel control of one vehicle (see Figure 1). Each wheel has three activated degrees of freedom (DOFs): driving, steering, and elevation (suspension of the vehicle). To control each DOF of the wheel, sensors and actuators as well as a control algorithm (in this case SWD, SWS, and ECS) are of vital importance. Thus every degree of freedom (mechanical part, sensors, actuators, control algorithms, and information processing) makes up one MFM.

The coupling of two or more MFMs produces an AMS. The AMSs include neither sensors nor actuators, and the mechanical couplings between two MFMs are purely passive ones. The AMS includes an energy supply for the actuators and sensors on the MFM level, e.g., a battery bank in a hybrid vehicle (Stolpe 1998). An example of the AMS level is also displayed in Figure 1. 
This 8-wheel vehicle can be a part of another mechatronic system. In this case, it is an agricultural machine. A machine of this kind is usually also equipped with a loader. Such a loader is made up of joints, each of them regarded as an MFM because it contains sensors, actuators, and local control. If any of these elements are coupled, the result will be another AMS.

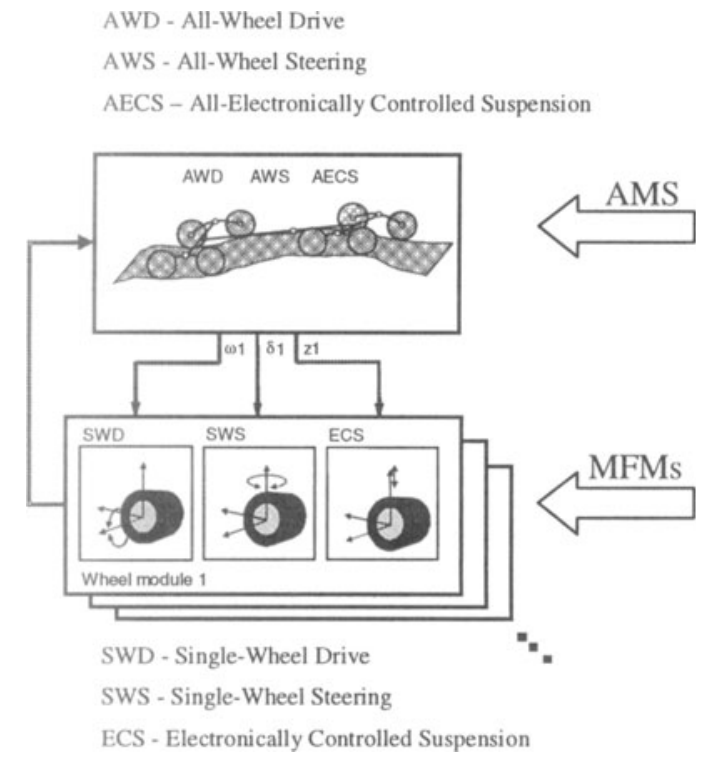

Figure 1. An example of MFMs and an AMS

The machine can be decomposed into three additional MFMs as follows:

1. engine: it supplies the oil pressure for the complete machine;

2. reference and visualization of the loader: they convey the reference values for the loader;

3. reference and visualization of the vehicle: they convey the reference values for the loader.

The main control (see Figure 2) is the result of the coupling of MFMs and AMSs. This is the AMS on the highest hierarchical level on the system.

The complete hierarchical decomposition of the system is shown in Figure 2. 


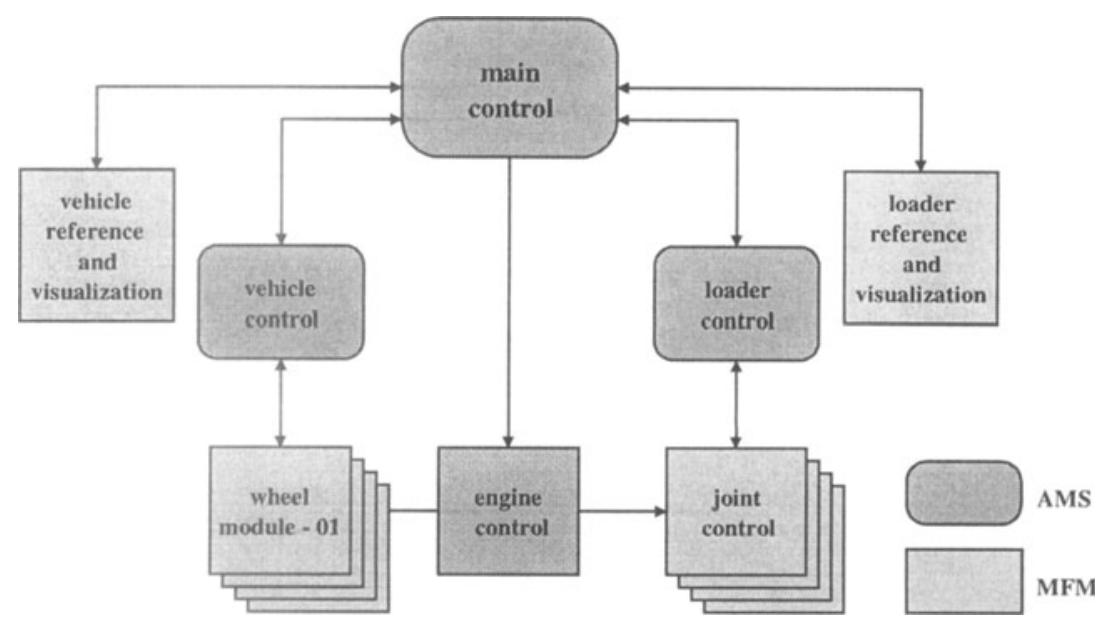

Figure 2. Hierarchical decomposition of an agricultural machine into MFMs and AMSs

\section{Hardware Description}

For this example approximately 139 sensor- and 50 actuator signals are estimated. The sensors and actuators for the Human-Machine Interface (HMI), generated from Loader reference and visualization and Vehicle reference and visualisation, are not included in the sum.

The hardware that will aggregate the complete machine during the design stages consists of 11 ECUs. The number of ECUs is based on the number of inputs/outputs of the system as well as the physical distribution of the sensors/actuators in the machine. Each ECU has 22 inputs ( 9 ADs from 0 to 5 volts, 10 DDs and 3 frequencymeasuring devices) and 9 outputs (5 PWMs and 4 DDs). With 11 ECUs, there are 242 inputs and 99 outputs available. To reduce message communication and respectively the bus load, 4 CANbuses are designed for the entire machine. On the basis of the control algorithm, the ECUs are distributed into four main subsets: loader, vehicle front, vehicle rear, and reference and visualization. For every pair of CANbuses, a TCAN (composed of 2 CAN controllers and one transputer) by ETAS (ETAS, 1997) will be used. The control algorithms will be implemented on USAPs (made up of one PowerPC and one transputer in communication via shared memory) also by ETAS (ETAS, 1996). In this configuration, the transputers links are responsible for the communication between the processors as well as that with the user. Figure 3 displays the projected hardware. 


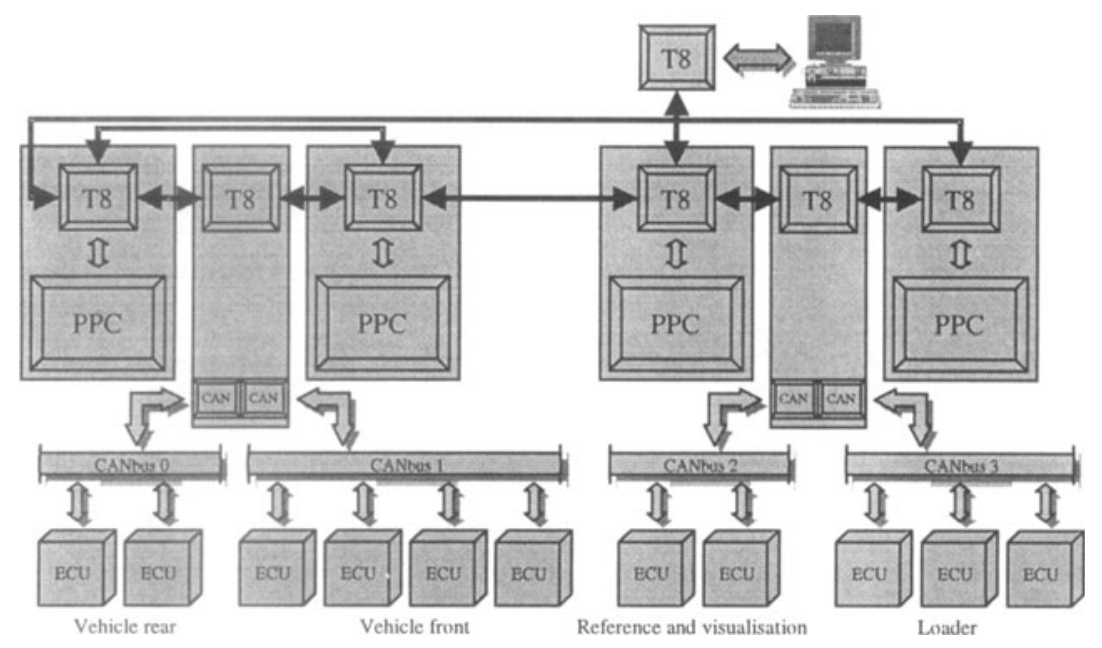

Figure 3. Hardware description for HILS of an agricultural machine

With the aim to perform a realistic simulation of CAN communication and signal propagation as well as to test the controller and the $\mathrm{I} / \mathrm{O}$ behaviour, a part of the entire hardware was tested in the laboratory. The test consisted of a suspension control of one wheel of the agricultural machine. The model and the control law were tested.

The hardware configuration employed for this simulation is shown in Figure 4. It consists on the right-hand side of a dSPACE C40 hardware with Matlab/Simulink and on the other side of a USAP and a T-CAN on an INMOS B008 with the IPANEMA (Stolpe 1998, Honekamp 1998) software platform. In-between are the ECUs.

A dSPACE C40 hardware was used for the simulation of the testbench dynamics, for the I/O of missing sensors/actuators, and the generation of test stimuli. At first we did an off-line simulation to evaluate the model and the control law of the wheel suspension. For this simulation we used only the dSPACE hardware. The model and the control law were described by Matlab/Simulink. The control law was programmed in one Simulink block.

The next step was to replace the control algorithm by a Simulink I/O block. The physical variables were converted in electric signals and sent to an ECU. This ECU sampled the signal and sent it to the USAP via CAN where the IPANEMA software platform was running at the moment. The control algorithm that was implemented by a calculator object from IPANEMA is the same as in the offline simulation. The output of the control was returned to the ECU via CAN and converted into a PWM signal. The dSPACE I/O read the signal and transferred it to the model. The minimal cycle time of the control was $10 \mathrm{~ms}$, due to the delay time of the ECU.

A connection panel was linked to the ECU and to the dSPACE hardware, thus allowing easy access to input and output signals. 


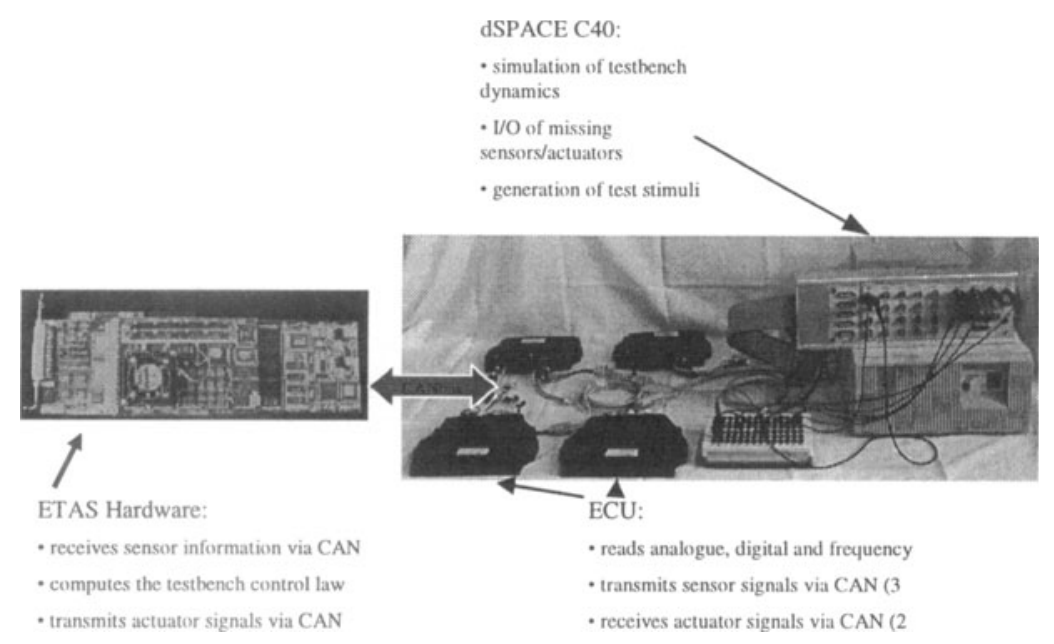

Figure 4. Hardware for the CAN and ECU tests in the laboratory

\section{HIL Simulation Platform}

IPANEMA (Integration Platform for Networked Mechatronic Applications) (Stolpe 1998, Honekamp 1998) can be used for a distributed HILS of a mechatronic system with the possibility of changing parameters and starting/stopping the complete system during simulation. The concept of MFMs and AMSs allows simple coupling and exchange of real elements for simulated elements. For highly complicated mechatronic systems, however, other functions gain ever more importance. One aspect is the possibility to handle errors during simulation. The following classes of errors may occur in a system:

1. Error from the simulation platform

Example: In the agricultural machine an error in the CAN communication could happen. This means that one process will wait for one message and not receive it. As a result, the other processes will wait in vain for a message from this particular process, and the entire system will become unstable. This is an effect of the hard connections between the control laws resp. the processes/processors.

2. Errors from a security system

Example: Due to security requirements, it is necessary for the operator to turn his chair to the front side to drive the machine. If the operator alters the position of the chair, an error will be generated and the error message sent to all processes responsible for the drive-motor control.

The error messages generated in both examples need not be sent to all processes/processors, just to a pre-defined group of them. A different communication level is necessary to support this feature.

Another important aspect is the possibility to implement the simulation with different step-times between the processes and their respective processors. For example, the 
step-time of the suspension control has to be shorter than that of the vehicle reference and visualization.

For other systems, e.g., an intelligent intersection management (Naumann 1997), a dynamic coupling of control processes (Sanden 1998) is required.

\subsection{Software Platform Description}

For a flexible HILS of the entire system, a software platform is currently being under way at MLaP. The main features of this platform are the following:

- possibility to connect the MFMs and AMSs like in the real system

- high performance of the system because of direct access to variables

- different levels of communication where it is possible to send a message to one processor node or to all processor nodes

- error messages on different levels

- different simulation rates, because each processor node has one real-time clock

- support of dynamic coupling

The Dispatcher/Worker model described in (Tanenbaum 1996) is used as a basis to control the processes on a processor and the communication with processes located on other processors.

The concept is made up of the following elements:

- Dispatcher: this element is the process manager on one processor. It is responsible for emitting the sequential order of processes that have to run. The dispatcher is also responsible for supervising the real-time on the processor (for more details, see next chapter). Starting and stopping of the simulation, parameter changes, communication and co-ordination of the dynamic coupling fall also into the dispatcher's responsibility.

- Worker: the description of the system and the data exchange with the real system fall into the worker's responsibility. There are two classes of workers:

- worker Model/Controller: here you find the equations that describe the dynamical behaviour or the control algorithm of the system. The C code is automatically generated from CAMeL tools (Homburg 1995).

- worker Adapter: gateway to the inputs and outputs of the system. The $\mathrm{C}$ code is automatically generated from ELBACO (Oberschelp 1998).

- Mailbox: The final element of the simulation platform is the mailbox. The mailbox is responsible for the communication with other MFMs, for the consistency of the variables, the composition/decomposition of messages, message-flow control and the coupling between the workers.

In this approach the system is non-preemptive, i.e., the workers cannot be interrupted during its run. Another novel feature of this system is a clock timer added to the model described in (Tanenbaum 1996) to indicate/set the step-time of the MFM (see Figure 6). Thus it is possible to have different time steps for each MFM.

The sequence of activated workers has to be pre-programmed in the dispatcher and cannot be altered during simulation. The dispatcher can also be programmed to run a worker more than once in a single time step. 


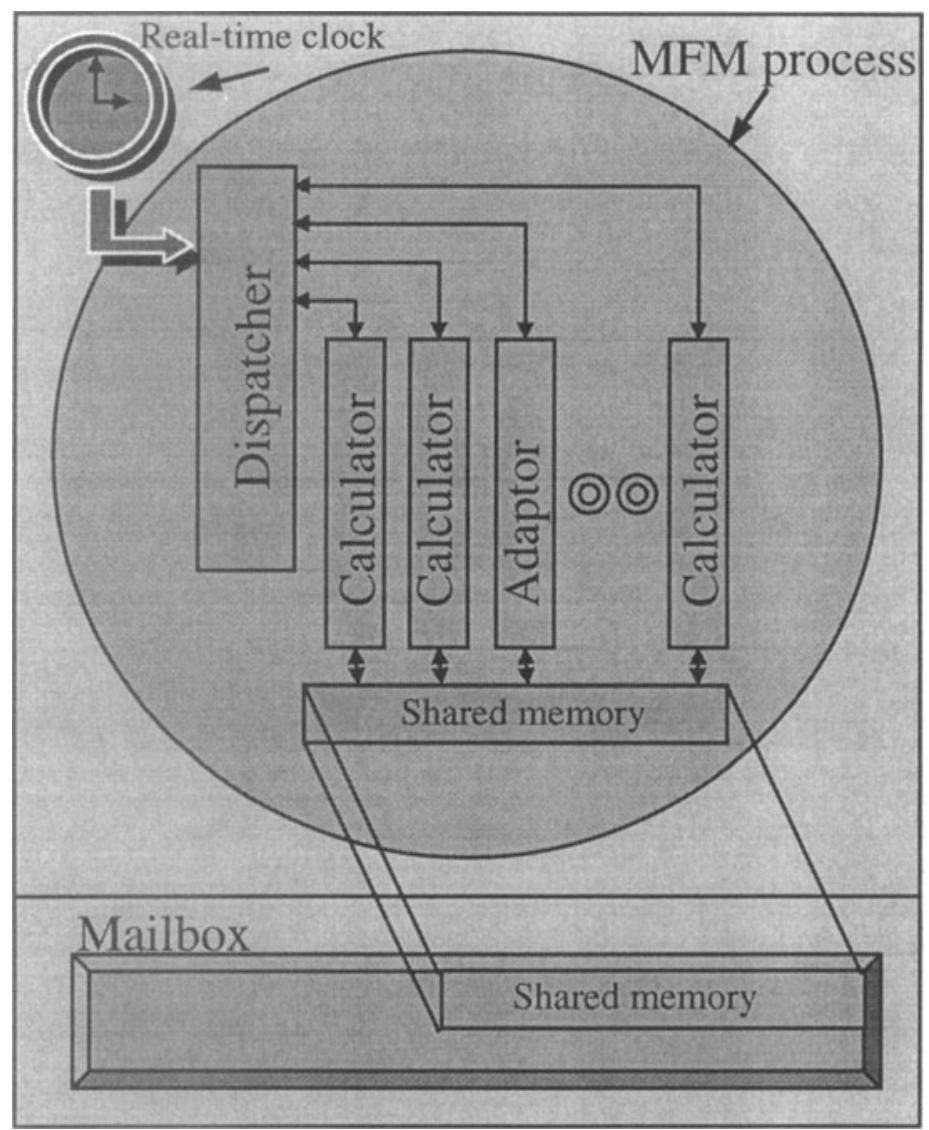

Figure 6. General approach to the HILS software platform

The number and types of workers in one MFM are not limited. A set of one dispatcher and multiple workers makes up an MFM that is encapsulated on one processor. The workers are based on the calculator and adaptor classes of IPANEMA.

\subsection{Steps for the HIL Simulation Software Platform}

This platform will be implemented at first on a USAP hardware. The objects (dispatcher, workers, and mailbox) can also run in parallel on this platform. In this hardware configuration, the mailbox and the dispatcher will run in parallel. They will be synchronized by means of messages transmitted via shared memory.

Implementation will be executed in three progressive stages:

1. Off-line simulation with one USAP - test of the dispatcher, worker and mailbox on a basic configuration of the platform without communication with other processors.

2. HILS with one USAP and one T-CAN - test of communication with another processor under real-time conditions. 
3. Distributed HILS with 2 USAPs and one T-CAN - test of distributed control algorithms and error-handling through error simulation (for example CAN communication).

The configuration of the last phase is illustrated in Figure 7.

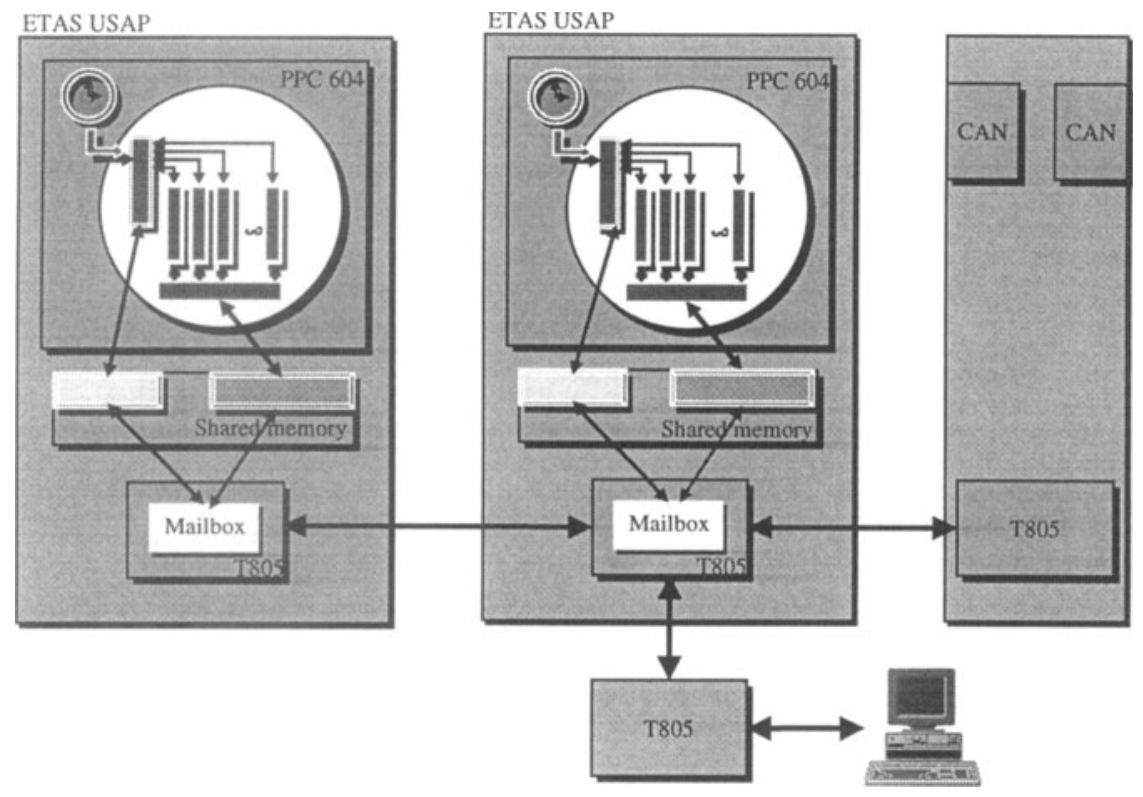

Figure 7. Hardware configuration of the HILS software tests

\section{End Notes}

A new approach to an HILS of a mechatronic system by distributed real-time information processing and a case study of an agricultural machine were presented. The division of the system elements is based on their respective functions (MFMs, AMS, and CMS).

The platform is based on a dispatcher/worker model. With this approach it is possible to connect subsystems the same way as in the real-system and to maintain the system hierarchy. It is also possible to alter the connections and the code of the workers. Another feature is the possibility of setting different time-steps for each MFM.

As an application, the example of an agricultural machine was presented. As far as hardware architecture is concerned, a PowerPC/Transputer combination was employed. Communication with the sensors is done via CANbus. The HIL simulation was used to test ECUs and CAN communication The concept presented is going to be implemented in a project to design a novel agricultural machine. 


\section{References}

ETAS GmbH \& Co. KG. (1996). Usap Transputer Module. ETA PC604-2000 V2 Documentation, Stuttgart, Germany.

ETAS GmbH \& Co. KG. (1997). TCAN V4.0 Transputer-CAN-Modul. Documentation, Stuttgart, Germany.

Homburg C. (1995). "Parallele Simulation mechatronischer Systeme auf der Basis von DSC." 10. Workshop Simulation verteilter Systeme und paralleler Prozesse. Dresden, Germany.

Honekamp U., Stolpe R., Naumann R., Lückel J. (1997). "Structuring Approach for Complex Mechatronic Systems." $30^{\text {th }}$ International Symposium on Automotive Technology \& Automation - Mechatronics/Automative Electronics, Florence, Italy.

Honekamp U. (1998). IPANEMA - Verteilte Echtzeit-Informationsverarbeitung in mechatronischen Systemen, Fortschr.-Ber. VDI, Reihe 20, Nr. 267. VDI-Verlag, Düsseldorf, Germany.

Oberschelp O. (1998). "Entwurf und Implementierung eines flexiblen Codegenerators zur Prozeßkopplung für verteilte Hardware-in-the-Loop Simulation.", Diplomarbeit, MLaP, Universität Paderborn, Germany.

Stolpe R., Zanella M. (1998). "A Distributed Hardware-in-the-Loop Simulation Environment in Use on a Testbed of a Series Hybrid Drive." ESM'98 - 12th European Simulation Multiconference, Manchester, England.

Tanenbaum, A.S. (1996). Distributed Operation Systems. Prentice-Hall, Englewood Cliffs, NJ. 